\title{
HEMODYNAMIC ALTERATIONS IN NORMOTENSIVE AND HYPERTENSIVE SUBJECTS DURING THE PYROGENIC REACTION
}

\author{
By STANLEY E. BRADLEY, HERBERT CHASIS, WILLIAM GOLDRING, AND \\ HOMER W. SMITH \\ (From the Departments of Physiology and Medicine, New York University College of Medicine, \\ and the Third (New York University) Division of Bellevue Hospital, New York City, \\ and the Department of Medicine, Boston University School of Medicine and \\ the Evans Memorial, Massachusetts Memorial Hospitals, Boston)
}

(Received for publication March 19, 1945)

It is well known that toxic substances, probably derivatives of bacterial protein (1), frequently appear in improperly distilled water. A dramatic and often potentially dangerous syndrome of chill and fever (pyrogenic reaction), occasionally complicated by peripheral circulatory collapse, follows the intravenous administration of solutions contaminated with these substances. A marked increase in renal blood flow (2), a reduction in arterial pressure (3) and an increase in cardiac output (4) have been observed during the pyrogenic reaction in normal man, indicating a marked reduction in over-all peripheral resistance. Three stages of arterial pressure change have been described (3) : namely, (a) a short period of elevated pressure during the chill and initial rise in temperature, $(b)$ a phase characterized by a moderate fall in blood pressure, coinciding approximately with the appearance of renal hyperemia, and (c) a marked reduction in blood pressure with a narrowing of the pulse pressure some 4 or 5 hours after the administration of the pyrogen. Although antipyretics, such as amidopyrine, when given in adequate doses prior to injection of the pyrogen, will prevent chill and fever, they do not affect renal hyperemia or phases $(b)$ and $(c)$. The reduction of arterial pressure $(c)$ is usually greater among hypertensive than among normal subjects and may be so profound as to result in a shocklike state, even in the absence of fever. It is not known whether the late reduction in blood pressure is a result of widespread vasodilation, reduced cardiac output or a combination of the two. Nor is it known whether there is any fundamental difference in response on the part of hypertensive as compared with normotensive individuals. The present study was undertaken to evaluate the hemodynamic factors involved in this reduction.

\section{METHODS}

Eight subjects, 6 of whom had essential hypertension, selected from the wards of the Third (New York University) Medical Division of Bellevue Hospital, and Evans Memorial Hospital (Boston University School of Medicine), were examined under basal conditions after several days of bed rest. All subjects were free of gross cardiac pathology.

Amidopyrine in doses of 0.6 gram was given to each subject every 4 hours for 5 or 6 doses during the 24 hours immediately prior to the administration of pyrogen. Typhoid vaccine and contaminated inulin (Pfanstiehl inulin lot No. 268) were used as sources of pyrogen. Pyrogenic inulin, in doses of 100 to $200 \mathrm{mgm}$. dissolved in sterile saline and passed through a Seitz filter, was administered intravenously to 4 of the hypertensive subjects (A. B., B. S., M. N., H. H.) while typhoid vaccine, in doses of $0.1 \mathrm{ml}$. (100 million organisms) was administered to 2 others (M. P., G. O.). Two normotensive subjects (P. H., S. V.) were given $300 \mathrm{mgm}$. and 150 mgm. of pyrogenic inulin, respectively. Control measurements were made prior to or within 20 minutes after the injection of pyrogen.

Cardiac output was measured with a modified Starr ballistocardiograph (5) using the wave area formula (6). Since ballistocardiograph complexes are abnormal in the majority of hypertensive subjects, only the results for subjects with normal complexes are reported in this study. All values for stroke volume and cardiac output have been corrected by the 18.5 per cent correction factor by which ballistocardiographic values have been shown to differ from those obtained by the direct Fick method (7). In 2 subjects (S. V., H. H.) cardiac output was determined by the direct Fick method following catheterization of the right auricle (8).

Arterial pressure in the femoral or brachial artery was recorded by arterial puncture and the Hamilton manometer (9) and mean pressure was computed planimetrically. A formula derived from Poiseuille's Law, based upon the output of the heart and the mean arterial pressure, was used in calculating the peripheral vascular resistance in absolute units (10).

Simultaneous renal function studies were carried out on 6 subjects (P. H., M. N., B. S., A. B., H. H., M. P.). Renal plasma flow was measured as the diodrast or paminohippurate clearance $(11,12)$, and glomerular filtration rate as the mannitol or inulin clearance $(12,13,14)$. 
RESULTS

\section{Normotensive subjects}

Circulatory adjustments during the afebrile pyrogenic reaction were studied in 2 normal convalescent male subjects (P. H., S. V.). Figure 1 illustrates the immediate response to pyrogenic inulin (300 $\mathrm{mgm}$.) seen in 1 of these individuals (P. H.). Peripheral resistance (R) decreased as renal plasma flow $\left(C_{D}\right)$ and cardiac output $(\mathrm{CI}-$ ballistocardiographic) increased. The output of the heart increased as a result of increases in both stroke volume (SV) and heart rate (HR). Arterial pressure (BP) showed a tendency to fall with a narrowing of the pulse pressure and at the termination of the study was definitely below the control level. No further depression of the blood pressure was noted and the subject was up and about several hours later without complaint.

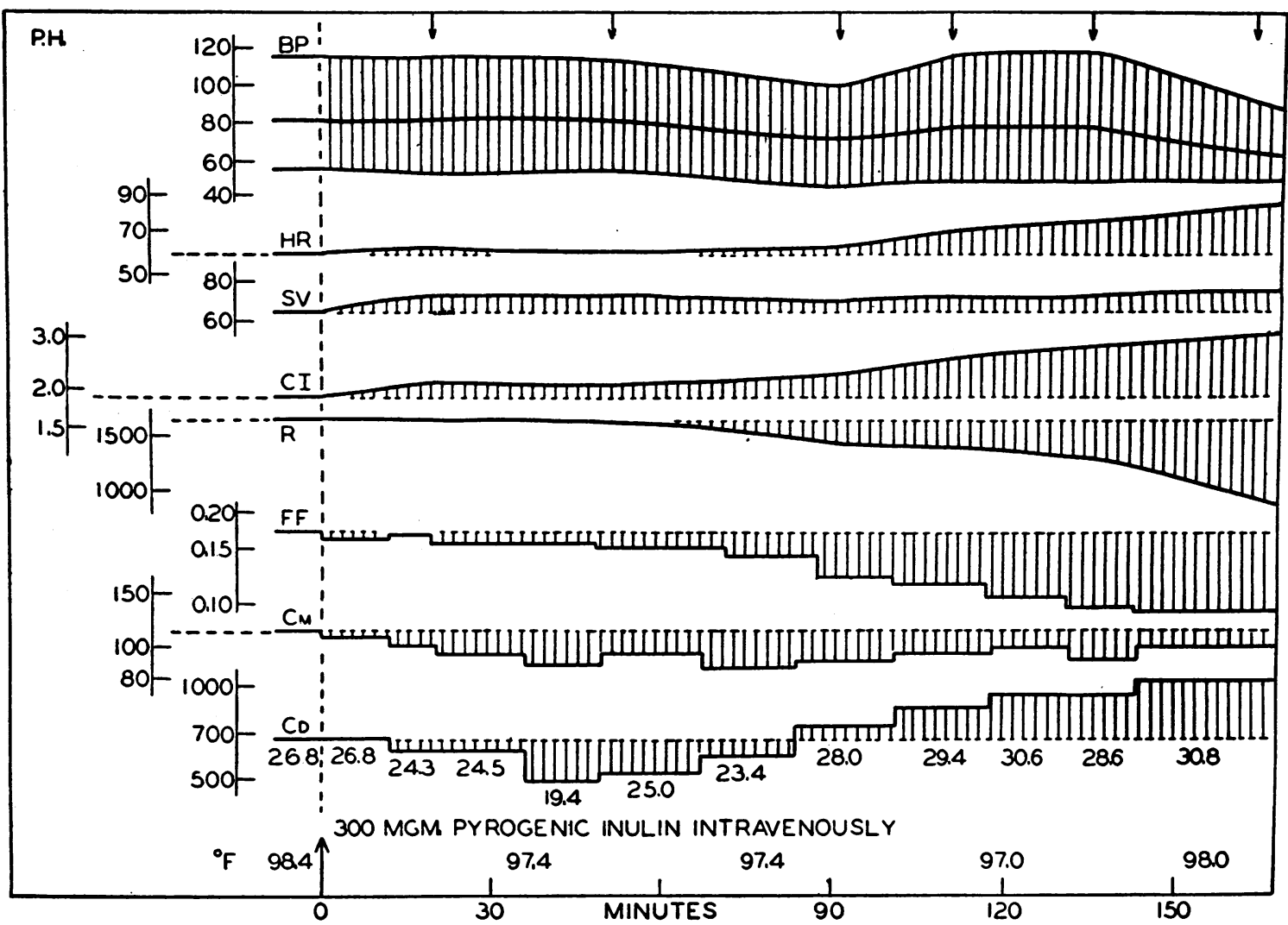

Fig. 1. Subject P. H. The Systemic and Renal Circulatory Effects of the Pyrogenic Reaction, Normal, Male, 52 Years

BP, blood pressure (Hamilton manometer) in $\mathrm{mm}$. $\mathrm{Hg}$, the thick line being the mean pressure; HR, heart rate; SV, stroke volume in $\mathrm{ml}$; $\mathrm{CI}$, cardiac index (ballistocardiograph) in liters per minute per square meter of body surface; $R$, peripheral resistance in dynes $\mathrm{cm}^{-6} \mathrm{sec}$.; FF, filtration fraction (fraction of renal plasma flow filtered at the glomerulus); $\mathrm{Cm}$, mannitol clearance (glomerular filtration rate in ml. per minute); $C_{D}$, diodrast clearance (renal plasma flow in $\mathrm{ml}$. per minute). Values of the renal fraction (per cent of the cardiac output passing through the kidneys) are inserted below each diodrast clearance period figure; ${ }^{\circ} \mathrm{F}$, rectal temperature in degrees of Fahrenheit. The arrows at the top of the figure indicate times at which hemodynamic data were obtained. All subjects described here were premedicated with amidopyrine.

Following the intravenous injection of $300 \mathrm{mgm}$. of pyrogenic inulin at zero time the cardiac output slowly rose and peripheral resistance decreased. The blood pressure fell from a control value of 116/58 to $90 / 50$, the mean pressure changing very little. The filtration rate decreased and the renal plasma flow, after falling during the first hour following injection of the pyrogen, increased markedly. The filtration fraction fell steadily. The blood pressure was well maintained despite the decrease in peripheral resistance and striking renal vasodilation.' This subject remained completely asymptomatic. 


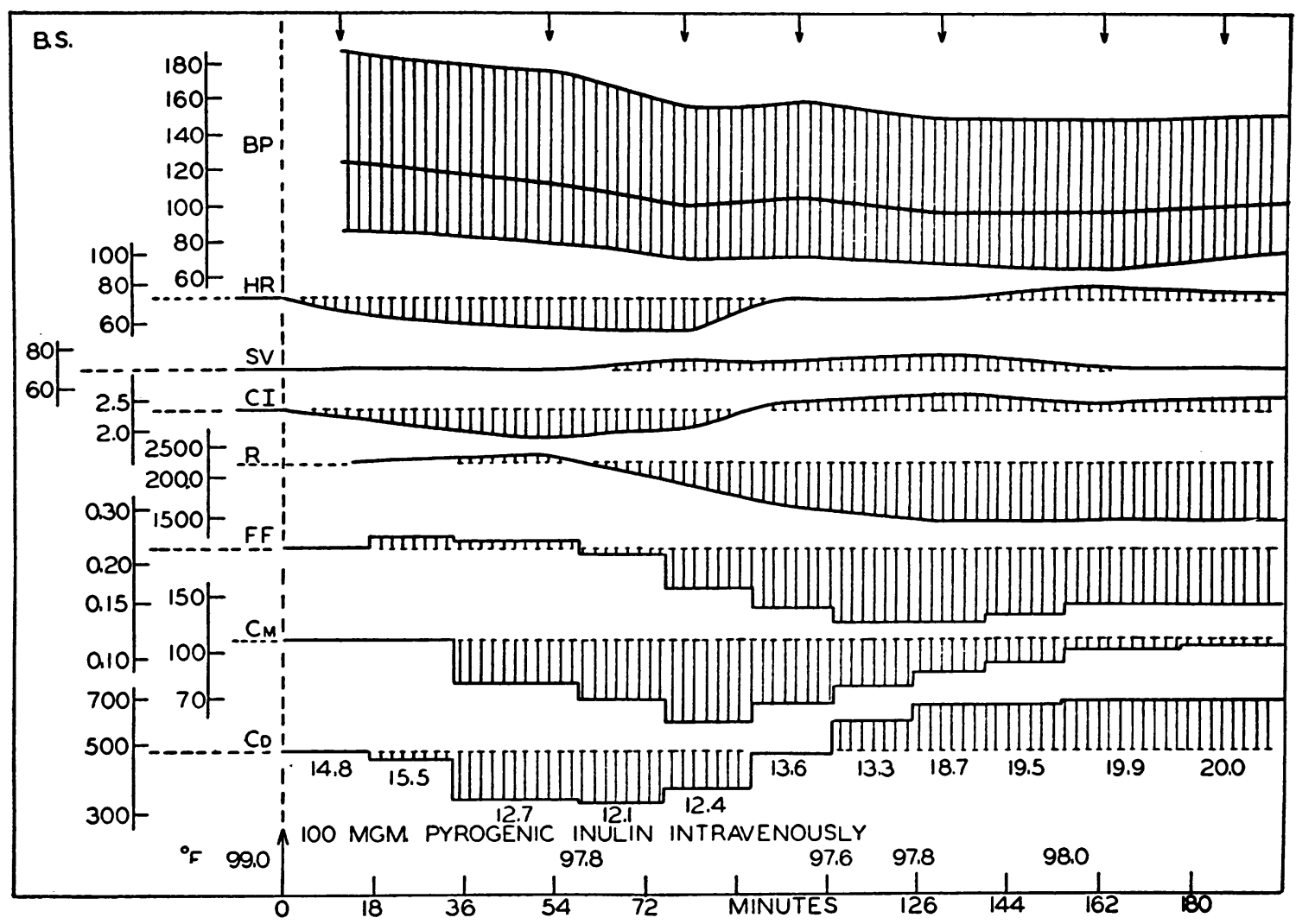

Fig. 2. Subject B. S. (see Figure 1 for symbols). Essential Hypertension, Female, 44 Years

Pyrogenic inulin (100 mgm.) was administered at zero time. The blood pressure fell from 185/84 to $150 / 70$, largely as a result of a decreased peripheral resistance. There was little change in cardiac output. The renal events were characteristic, except that a marked decrease in glomerular filtration rate was observed during the first 2 hours.

A second normal subject (S. V.) was given $150 \mathrm{mgm}$. of pyrogenic inulin. Cardiac output, measured by both the direct Fick method and ballistocardiograph, increased by 54 per cent and 64 per cent, respectively, over a period of 3 hours following administration of pyrogen. Arterial pressure showed no significant change and, though followed for several hours, no trend toward hypotensive levels was noted. The peripheral resistance fell as cardiac output increased. Renal function was not measured in this patient. On return to the ward, he became ambulatory immediately without ill effect. ${ }^{1}$

1 The cardiovascular changes during the afebrile pyrogen reaction were qualitatively similar in these 2 patients regardless of the method employed in determining cardiac output. However, a comparison (S. V.) of the values of cardiac output obtained by the 2 methods revealed a striking discrepancy at the height of the pyrogenic reaction. The control values of cardiac index agreed fairly well,
Since the findings of increased cardiac output, decreased peripheral resistance and fall in blood pressure in these 2 subjects agreed with those of other workers $(3,2,4)$ and since these responses were typical of the many pyrogenic reactions we have observed in normal man, it was felt that further control studies were unnecessary.

\section{Hypertensive subjects}

In 2 of 6 hypertensive subjects (B. S., M. P.) the response to pyrogen was essentially similar to

the direct Fick method giving a figure of 4.221 . per min. per $\mathrm{m}^{2}$, the ballistocardiograph (BCG) 3.941 . per min. per $\mathrm{m}^{2}$. Following the injection of pyrogen the cardiac index was increased to 5.071 . per min. per $\mathrm{m}^{2}$ (BCG) and 6.9 (direct Fick). The discrepancy indicates that caution must be exercised in accepting absolute values for cardiac output determined by the ballistocardiograph during unusual physiological conditions. The above error, however, tends to minimize apparent increases in cardiac output and decreases in peripheral resistance. 
that of the normotensives described above. The hemodynamic changes observed in 1 of these (B. S.) are illustrated in Figure 2. A gradual fall in arterial pressure without much change in pulse pressure followed the injection of pyrogenic inulin. Cardiac output, pulse rate and renal plasma flow increased, while a significant and progressive diminution of the peripheral resistance was noted. Blood pressure levels were not measured following completion of the illustrated part of the study; however, the patient remained in bed and asymptomatic. Abnormalities of the ballistocardiographic record made measurement of the cardiac output impossible in subject M. P., a young female with hypertension of at least 2 years' duration. However, as among the normal subjects, no significant change in arterial pressure was observed, following the administration of typhoid vaccine although the reaction elicited renal hyperemia associated with a decreased filtration fraction. In one respect this subject responded in an abnormal manner. At the peak of the pyrogenic reaction, as revealed by renal hyperemia, it was found that assumption of the upright position resulted in the rapid onset of dizziness and faintness associated with tachycardia. Unfortunately, it was impossible to obtain a record of blood pressure at this time. Return to the horizontal position quickly relieved the symptoms and the subject remained asymptomatic, with an unchanged

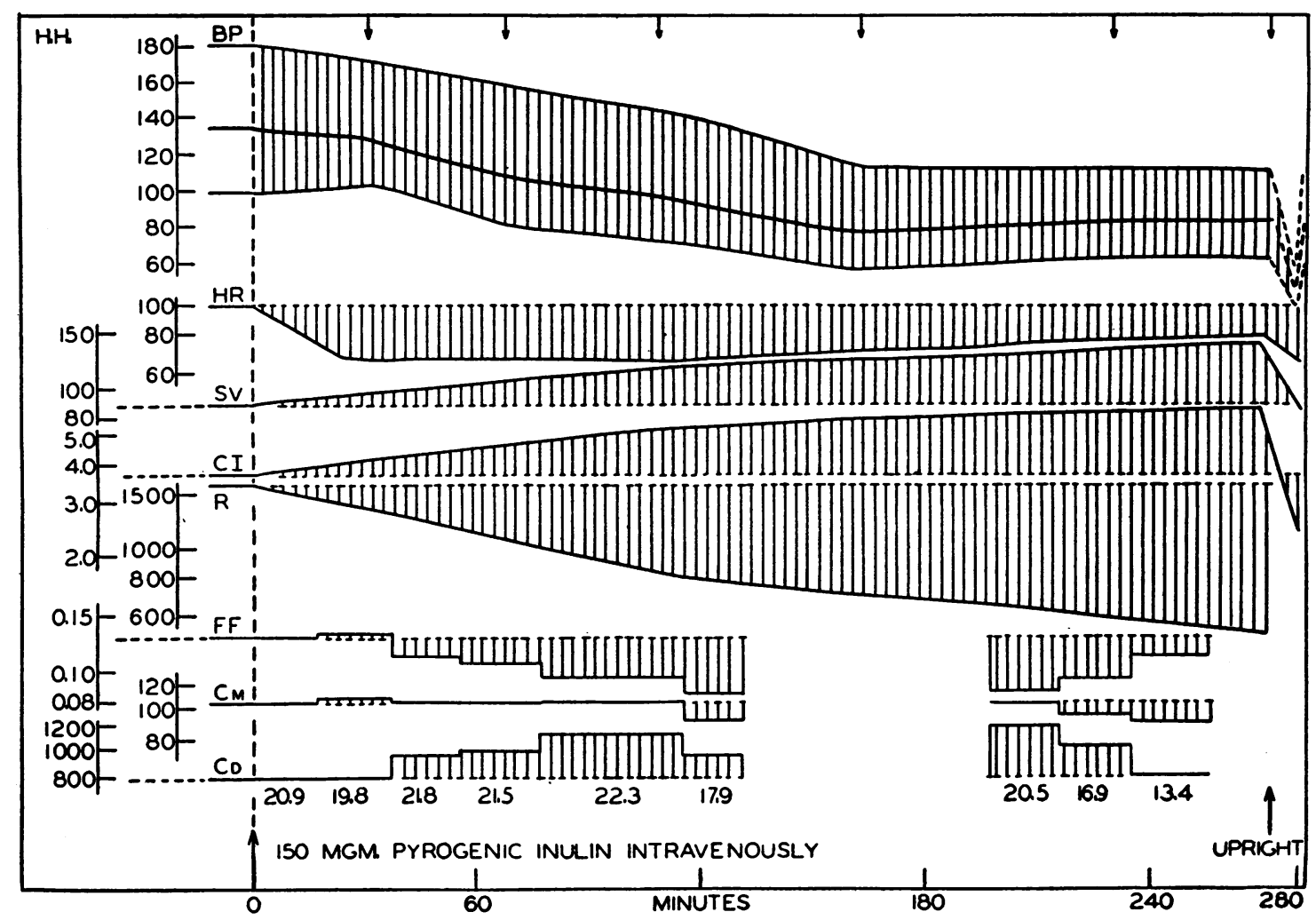

Fig. 3. Subject H. H. (CI, Cardiac Output in Liters per Minute per Square Meter of Body Surface Measured by Direct Fick Method; Symbols Otherwise as Figure 1.) Essential HypertenSion, Male, 49 Years

Following administration of $150 \mathrm{mgm}$. of pyrogenic inulin the cardiac output steadily increased as a result of an increased stroke volume, while the peripheral resistance fell. Blood pressure fell over a period of 3 hours to normotensive levels where it was well maintained by reciprocal changes in cardiac output and peripheral resistance. Renal plasma flow increased during the first 4 hours and then returned to the control level. There was very little change in the filtration rate and the filtration fraction decreased. At upright, the subject was placed in a sitting position and hypotension (interrupted pressure curve indicates sphygmomanometric measurements) developed associated with a marked fall in cardiac output. Recovery followed the return to recumbency. Body temperature, not recorded here, remained constant throughout. 


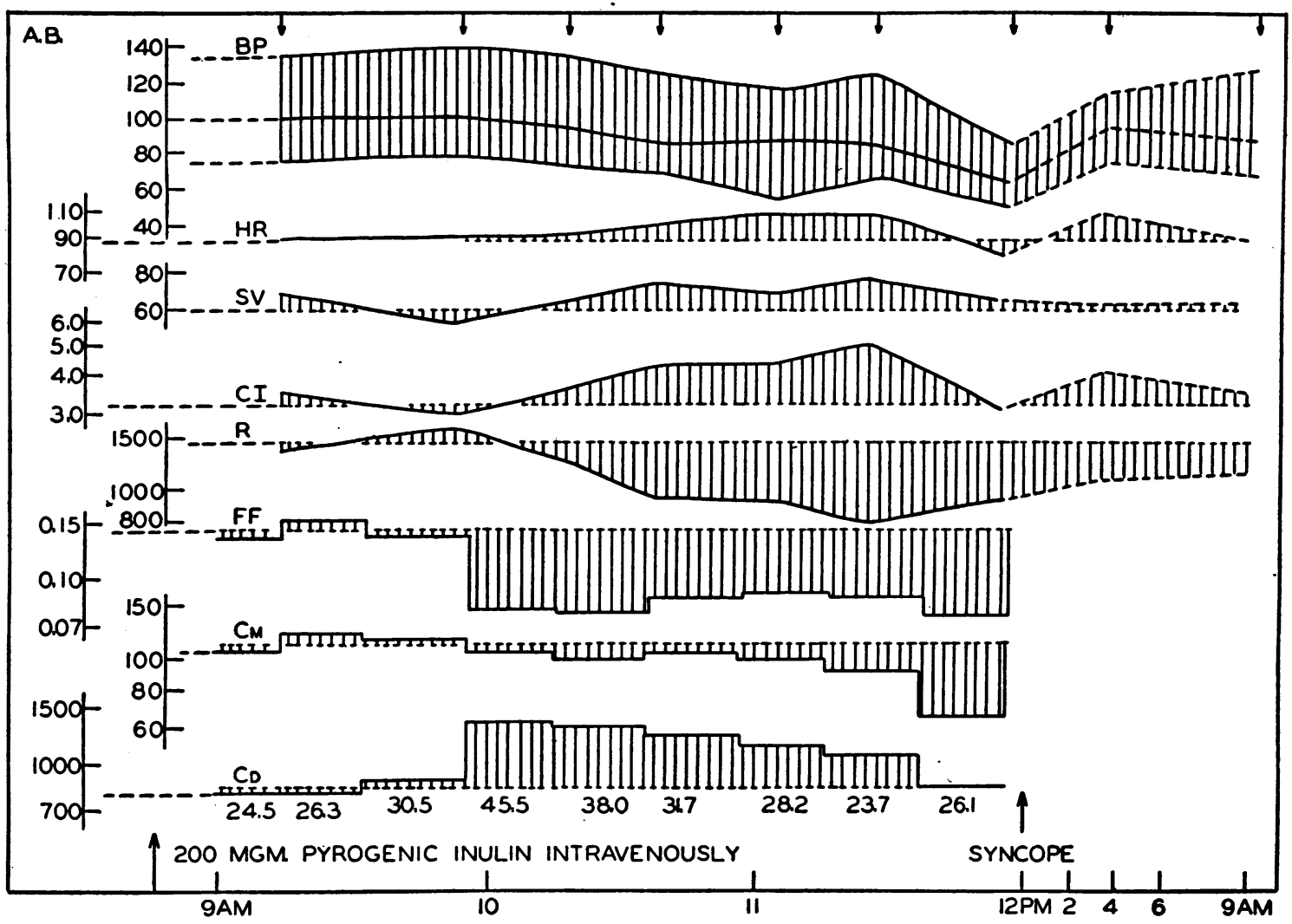

Fig. 4. Subject A. B. Essential Hypertension, Male, 33. (See Figure 1 for Symbols)

The blood pressure fell very little during the first 3 hours following the administration of $200 \mathrm{mgm}$. of pyrogenic inulin. The decrease in peripheral resistance during this period was balanced by an increase in cardiac output. At 12:00 p.m., the renal function studies were completed and the patient rose to his feet. Very shortly thereafter syncope occurred and blood pressure could not be obtained. Interrupted pressure curve marks sphygmomanometric measurements. It should be noted that cardiac output and pulse rate decreased just prior to the onset of syncope. Body temperature, not recorded here, was normal throughout.

blood pressure as long as she did not sit or stand up. This abnormal response to postural change cleared slowly during the following 12 hours.

In the remaining subjects (H. H., A. B., M. N., G. O.) the evidence of maladjustment to postural change was clearly demonstrated. In every case the assumption of the standing or sitting position was followed by hypotension and faintness or syncope several hours after injection of pyrogen. In 1 (G. O.) impending syncope was present in the horizontal position. These subjects will be described in order of the severity of the hypotensive response.

Subject H. H. (Figure 3) showed a typical response. The cardiac output (direct Fick) increased markedly, the peripheral resistance decreased and the blood pressure fell slowly to a normal level. In the late afternoon, after the termination of the renal studies and more than 4 hours after pyrogen had been administered, he was raised to a sitting position. At this time the blood pressure had fallen to normotensive levels, cardiac output had increased and the peripheral resistance had fallen to a markedly low level. On sitting up, the blood pressure (measured sphygmomanometrically) fell almost immediately to a level of $60 / 40$ and bradycardia appeared. A direct Fick determination was made immediately (in recumbency) and the cardiac output was found to have fallen well below the control values. On completion of the Fick measurement, arterial pressure as determined by the Hamilton manometer had returned to the level existing before the subject was elevated. It was found that the pressure fell rapidly each time he was brought to the sitting position, with rapid restoration on assum- 
ing the supine posture. No attempt was made to calculate the peripheral resistance during these tests because of the difficulty of measuring cardiac output by the direct Fick method and determining the arterial pressure simultaneously.

Subject A. B. (Figure 4) was a 33-year-old male with essential hypertension of at least 2 years' duration. His blood pressure fluctuated widely, falling to normal levels on bed rest. Following administration of pyrogen, the arterial pressure fell slowly with a narrowing of the pulse pressure. As renal hyperemia appeared the cardiac output, stroke volume, and pulse rate increased with a significant diminution of peripheral resistance. At the termination of the renal function study a marked drop in the blood pressure associated with a fall in cardiac output was noted. A few minutes later, on rising to his feet, the subject suddenly became pulseless and fainted. Several hours later the blood pressure was found somewhat higher than just prior to syncope and the cardiac output was again above control values. The peripheral resistance continued to be depressed. The following morning the blood pressure and cardiac output had returned to the control level.

Figure 5 illustrates a similar reaction observed in another young hypertensive, M. N. She was examined again 3 hours after the termination of the renal function study and by this time was found to have a markedly reduced arterial pressure and pulse pressure associated with a falling cardiac output. Soon after she was placed in a wheel chair to return to the ward, she suddenly became pulseless and complained of faintness. This marked hypotensive state lasted for several

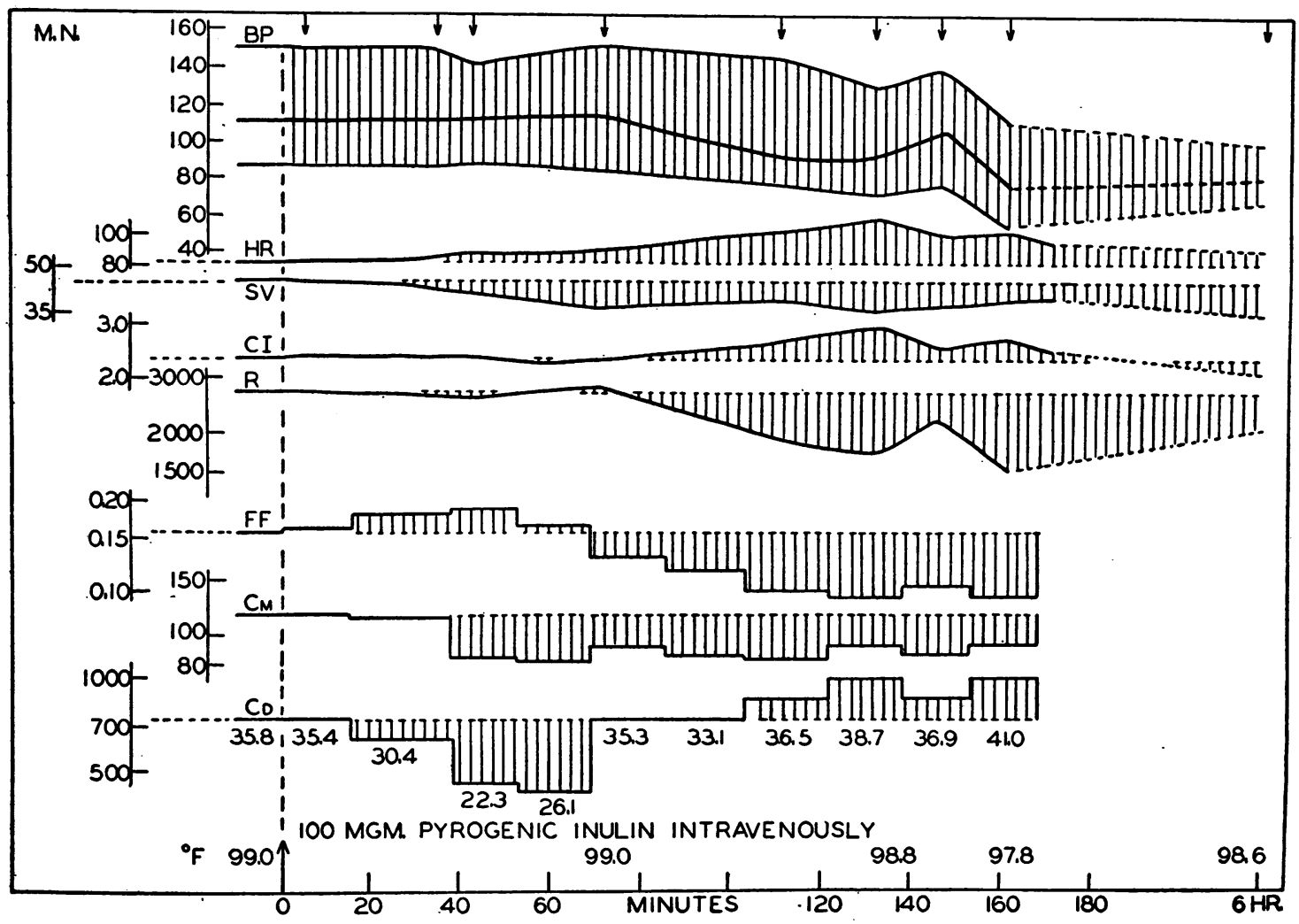

Fig. 5. Subject M. N. Essential Hypertension, Female, 20. (See Figure 1 for Symbols)

The arterial pressure changed very little during the first 2 hours after the administration of $100 \mathrm{mgm}$. of pyrogenic inulin. Peripheral resistance fell while cardiac output increased. Six hours after the pyrogen was administered it was found that the arterial pressure (interrupted pressure curves denote sphygmomanometric measurements) had fallen to hypotensive levels, associated with a decrease in cardiac output below the control value. The peripheral resistance remained reduced. Shortly after this measurement was made, the subject became pale, pulseless and dizzy. She remained so for several hours. 


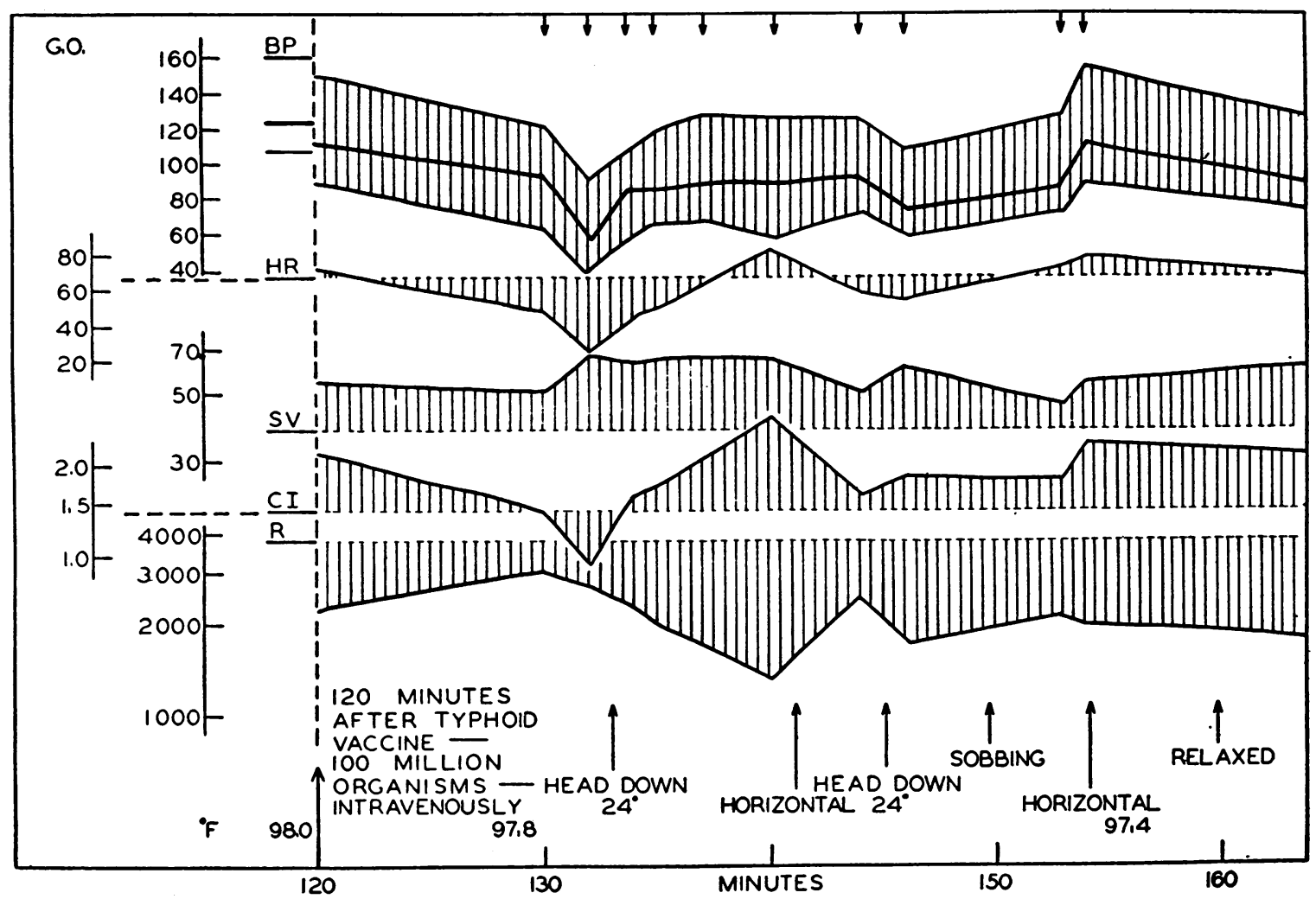

Fig. 6. Subject G. O. Essential Hypertension, Female, 44. (Symbols Same as Figure 1)

No hemodynamic change other than a falling peripheral resistance and an increasing cardiac output was noted during the first 2 hours after pyrogen (typhoid vaccine) was administered. During the next 10 minutes, however, the blood pressure fell to a normotensive level and then fell precipitously to $92 / 40 \mathrm{~mm}$. $\mathrm{Hg}$ associated with a parallel decrease in cardiac output. The peripheral resistance increased slightly. Restoration to a normotensive level followed a change of position from recumbency to $24^{\circ}$ head down. The recovery was associated with an increased cardiac output and a further fall in peripheral resistance. The return to the horizontal position brought about a similar sequence of events and the head down position was necessary to avert syncope and relieve faintness. At 150 minutes the patient was questioned about a relative whom she was known to dislike, and she broke into tears and sobbing and shortly thereafter it was found that the blood pressure had returned to a hypertensive level, She was again placed in the horizontal position and the pressure slowly returned to normotensive levels where it remained for approximately 24 hours.

hours associated with faintness, nausea and vomiting, marked pallor and cold extremities.

Subject G. O. (Figure 6) was a 44-year-old female with a well-established hypertension of 3 years' known duration. For 2 hours following administration of pyrogen the blood pressure was well sustained and cardiac output increased while peripheral resistance slowly fell. Then, while still in the horizontal position, a drop in blood pressure to normotensive levels occurred followed by a further rapid fall of systolic pressure to $90 \mathrm{~mm}$. $\mathrm{Hg}$ and diastolic to $40 \mathrm{~mm}$. $\mathrm{Hg}$, associated with marked bradycardia, faintness; and nausea. Although peripheral resistance increased somewhat during the pressure fall, the most striking hemodynamic change was the marked fall in cardiac output. When the subject was placed in the shock position, the blood pressure returned to normotensive levels, the cardiac output increased as a result of an increased heart rate and a larger stroke volume, with subjective improvement. On the return to the horizontal, circulatory changes similar to those seen during the initial episode rapidly recurred. Again the shock position resulted in improvement. At this time the subject was questioned concerning a member of her family against whom she was known to harbor a strong grievance and a stormy emotional response was 
elicited. The blood pressure rose quickly to a hypertensive level apparently as a result of an increase in cardiac output due to an increased heart rate and increased stroke volume, the peripheral resistance showing little change. Following the return to horizontal the pressure slowly returned to a normotensive level where it remained for approximately 18 hours. During most of this time the subject was maintained in shock position to assure maximal comfort.

\section{Renal fraction}

The percentage of the cardiac output diverted to the kidneys (renal fraction) was calculated in every instance in which simultaneous measurement of renal blood flow and cardiac output was made. The figures are inserted below the graphic record of the diodrast clearances in Figures 1 to 5. The renal fraction increased in every instance over the control figure, usually reaching a maximum at the peak of renal hyperemia. On 3 (P. H., B. S., M. N.) of the 5 occasions in which the renal fraction was measured it fell simultaneously with the renal blood flow shortly before the appearance of renal hyperemia. This phase of decreased renal fraction and renal blood flow was usually associated with a reduction in the filtration rate without much change in the filtration fraction. These phenomena appear to be independent of hypertensive disease and, though not consistently present, appear frequently enough to be noteworthy (2).

\section{DISCUSSION}

It is clear from this study that peripheral vasodilation is the primary hemodynamic change in both normal and hypertensive subjects during the pyrogenic reaction. Qualitatively the 2 groups behaved similarly, but there appear to be quantitative differences in the hemodynamic alteration and in the extent to which adjustment is possible. In normal subjects and in some hypertensives, augmentation of cardiac output compensates for the decrease in peripheral resistance and arterial pressure is maintained. But in some hypertensive subjects $(H . H$. at the beginning and G. O. at the close of the study) the arterial pressure fell to normotensive levels and was thereafter maintained constant. In 1 hypertensive subject (G. O.), ad- justment failed completely and the blood pressure fell to an alarmingly low level. In every instance the fall in blood pressure could be attributed to the failure of cardiac output to increase in proportion to the dilatation of the peripheral vascular bed.

Hypertensive subjects, as a group, appear to be particularly vulnerable to conditions demanding excessive circulatory adjustments. It is well known (15) that orthostasis is associated with reflex vascular changes by which return of blood to the heart is sustained in spite of gravitational pooling of blood in the extremities. It is presumed that the essential mechanisms involved in these adjustments are much the same as in phases of circulatory inadequacy induced by pyrogen. During the pyrogenic reaction in normal subjects, these adjustments appeared to be adequate (P. H., S. V.) but among hypertensive subjects orthostasis was not efficiently compensated, cardiac output decreased excessively and, as a result, hypotension and syncope ensued. Improvement of return of blood to the heart, by placing the subject in the shock position or by the maneuvers of sobbing, as in patient G. O., resulted in prompt alleviation of symptoms and increase in intraarterial pressure. It should be noted, however, that the arterial pressure never returned to the control, hypertensive levels. Marked hypotension apparently never followed further decrease in peripheral resistance, but was always attributable to a fall in cardiac output superimposed upon an already dilated vascular bed. As a matter of fact, in G. O. (Figure 6) a compensatory increase in the peripheral vascular resistance was apparent at a time when arterial pressure was falling precipitously. ${ }^{2}$

Consequently, it seems logical to assign the cause of the hypotension that occurs in hypertensive subjects during the pyrogenic reaction, whatever the precipitating factor, to a disorder of the mechanisms that effect compensatory increases in cardiac output. The increases in cardiac output

2 This change in calculated resistance may have resulted from inaccuracies inherent in the ballistocardiographic method of determining cardiac output, for an erroneously low value of cardiac output would produce such an apparent elevation of calculated resistance. The behavior of the peripheral resistance in this situation requires further study with a more precise method for measuring cardiac output. 
demanded to maintain pressure levels in our subjects were by no means in excess of daily requirements (16) and in none of these subjects was there any evidence of diminished cardiac reserve. It is likely, therefore, that cardiac output would have been efficiently sustained had an adequate return and filling mechanism been operative. This view is strengthened by the favorable response to measures that improved cardiac filling. In the hypotension of spinal anesthesia it is probable that relaxation of the muscles of the lower extremities interferes with activity of the "booster pump" system of the intramuscular channels and so contributes to a reduction in cardiac output (17), but this factor cannot be evoked to explain the defective return of blood to the heart during the pyrogenic reaction. Moreover, no change in respiratory activity was apparent as a possible cause of an altered gradient of venous pressure between the thorax and the abdomen. It seems more likely that capillary and venous dilatation and inadequate adjustment to postural change were at fault in the studies reported here. Such a dilatation might be expected to pool a large quantity of blood in the lower extremities and abdominal cavity in the upright position or even in recumbency. It is noteworthy that these mechanisms appear to be altered by hypertensive disease, usually considered pre-eminently a disorder of the arterial and arteriolar channels of the circulatory system.

It is probable that the fall in peripheral resistance was a result of arteriolar dilatation, but whether this vasodilation is generalized or confined to particular portions of the vascular bed is undetermined. No change in the blood flow to the skin or skeletal muscle has been noted in normal man during the afebrile pyrogen reaction (18), while it is known that under these conditions the renal blood flow is increased (2) and it is here demonstrated that this increase represents an actual increase in the fraction of the total blood put out by the heart, demonstrating that renal vasodilatation was proportionately greater than dilatation elsewhere in the vascular bed. Hence, the kidney appears to be peculiarly susceptible to the action of pyrogen in both normal and hypertensive individuals.

It has been noted, and confirmed here, that the renal blood flow frequently decreases shortly after the administration of pyrogen (2). This phenomenon occurs at the time when the chill usually develops in the unpremedicated subject. These workers (3) noted an increase of arterial pressure in unpremedicated subjects during the chill and considered this as indicative of an overall vasoconstriction. However, no vasoconstriction was evident in the values for peripheral resistance or in the arterial pressure in the absence of the chill and fever either in previous studies (2) or in the present series. Nevertheless, the decrease in renal blood flow so often associated with a fall in renal fraction during the early or latent period denotes the presence of vasoconstriction in the kidney, a phenomenon which presumably is associated with vasodilatation elsewhere since it is not reflected in any change of total peripheral resistance or mean arterial pressure. The identity of the opposing dilated vascular beds remains obscure. The failure of the filtration fraction to show any consistent change during the vasoconstrictor phase is evidence that both afferent and efferent glomerular arterioles contribute to the reduction in size of the renal vascular bed.

\section{SUMMARY}

1. A study of the hemodynamic changes induced by the afebrile pyrogenic reaction (in premedicated subjects) has been made in 2 normal and 6 hypertensive persons.

2. In every instance cardiac output increased as a result of an increase in both pulse rate and stroke volume, while total peripheral resistance decreased.

3. In normal subjects and in some hypertensive individuals reciprocal changes in peripheral resistance and cardiac output resulted in adequate maintenance of arterial pressures. In 2 hypertensive subjects, however, arterial pressure fell to normotensive levels and in a third, marked hypotension developed. In the latter, a change to head-down position was necessary to maintain the blood pressure at physiologically adequate levels.

4. Normotensive subjects are capable of adequate vascular adjustment to postural change during the pyrogenic reaction. Among hypertensives, however, even though adjustment may be adequate in the recumbent position, orthostasis may 
result in a shock-like state. The observations reported here indicate that a fall in cardiac output is responsible for circulatory inadequacy under these conditions.

5. Renal hyperemia occurred in each instance studied and in the 5 subjects in whom cardiac output and renal blood flow were measured simultaneously, the renal fraction increased, implying relatively greater vasodilation in the renal vascular bed than in the rest of the circulatory system.

This work was aided by a grant from The Commonwealth Fund.

We are indebted to Dr. André Cournand, Dr. R. L. Riley, and Dr. E. S. Breed for the measurement of the cardiac output by the direct Fick method, to Dr. R. W. Wilkins and Dr. G. P. Bradley for assistance in conducting certain of the studies, to Mr. Ray Houde for aid in computations, and to Miss Claire Lawler, Miss Martha Barrett, and Miss Constance Qua for technical assistance.

\section{BIBLIOGRAPHY}

1. Seibert, F. B., The cause of many febrile reactions following intravenous injections. I. Am. J. Physiol., 1925, 71, 621.

2. Chasis, H., Ranges, H. A., Goldring, W., and Smith, H. W., The control of renal blood flow and glomerular filtration in normal man. J. Clin. Invest., 1938, 17, 683.

3. Chasis, H., Goldring, W., and Smith, H. W., Reduction of blood pressure associated with the pyrogenic reaction in hypertensive subjects. J. Clin. Invest., 1942, 21, 369.

4. Grollman, A., Variations in the cardiac output of man. V. The cardiac output of man during the malaise and pyrexia following the injection of typhoid vaccine. J. Clin. Invest., 1929, 8, 25.

5. Starr, I., Rawson, A. J., Schroeder, H. A., and Joseph, N. R., Studies on the estimation of cardiac output in man, and of abnormalities in cardiac function, from the heart's recoil and the blood's impacts; the ballistocardiogram. Am. J. Physiol., 1939, $127,1$.

6. Starr, I., and Schroeder, H. A., Ballistocardiogram.
II. Normal standards, abnormalities commonly found in diseases of the heart and circulation, and their significance. J. Clin. Invest., 1940, 19, 437.

7. Cournand, A., Ranges, H. A., and Riley, R. L., Comparison of results of the normal ballistocardiogram and a direct Fick method in measuring the cardiac output in man. J. Clin. Invest., 1942, 21, 287.

8. Cournand, A., and Ranges, H. A., Catheterization of the right auricle in man. Proc. Soc. Exper. Biol. and Med., 1941, 46, 462.

9. Hamilton, W. F., Brewer, G., and Brotman, I., Pressure pulse contours in the intact animal. I. Analytical description of a new high-frequency hypodermic manometer with illustrative curves of simultaneous arterial and intracardiac pressures. Am. J. Physiol., 1934, 107, 427.

10. Apéria, A., Hemodynamical studies. Skand. Arch. f. Physiol., 1940, Suppl. 16, 83, 1.

11. Smith, H. W., Finkelstein, N., Aliminosa, L., Crawford, B., and Graber, M., The renal clearances of substituted hippuric acid derivatives and other aromatic acids in dog and man. J. Clin. Invest., 1945, 24, 388.

12. Smith, H. W., Goldring, W., and Chasis, H., The measurement of the tubular excretory mass, effective blood flow and filtration rate in the normal human kidney. J. Clin. Invest., 1938, 17, 263.

13. Smith, W. W., Finkelstein, N., and Smith, H. W., Renal excretion of hexitols (sorbitol, mannitol, and dulcitol) and their derivatives (sorbitan, isomannide, and sorbide) and of endogenous creatinine-like chromogen in dog and man. J. Biol. Chem., 1940, 135, 231.

14. Goldring, W., and Chasis, H., Hypertension and $\mathrm{Hy}-$ pertensive Disease. The Commonwealth Fund, New York, 1944, p. 196.

15. Hellebrandt, F. A., and Franseen, E. B., Physiological study of the vertical stance of man. Physiol. Rev., 1943, 23, 220.

16. Grollman, A., The Cardiac Output of Man in Health and Disease. Charles C. Thomas, Baltimore, 1932.

17. Rovenstine, E. A., Papper, E. M., and Bradley, S. E., Circulatory adjustments during spinal anesthesia in normal man with special reference to the autonomy of arteriolar tone. Anesthesiology, 1942, 3, 421.

18. Bradley, S. E., and Friedland, C., Unpublished data. 